\title{
Retour d'expérience des six évacuations sanitaires aériennes collectives MoRPHEE durant la pandémie de Covid-19
}

\author{
Feedback from the Six Collective Medical Evacuation Missions MoRPHEE during \\ the COVID-19 Pandemic
}

\author{
M. Beaussac $\cdot$ B. Distinguin $\cdot$ J. Turc $\cdot$ M. Boutonnet \\ Reçu le 24 juin 2020; accepté le 30 juin 2020 \\ (C) SFMU et Lavoisier SAS 2020
}

Résumé Après son apparition en Chine à la fin de 2019, l'épidémie de coronavirus 2019 (Covid-19) a rapidement provoqué le risque d'une saturation des ressources en soins intensifs dans chaque pays touché. La répartition de la maladie entre les différents territoires est hétérogène. Le transport interhospitalier sur longue distance de patients atteints de Covid-19 dans le but de limiter la pression sur les unités de soins intensifs au niveau national ou international n'a pas encore été décrit. L'objectif de cet article était de fournir des données descriptives des six missions d'évacuation aéromédicale collective (Evasan) de patients atteints de Covid-19 réalisées en Europe et sur le territoire national français les 18, 21, 24, 27, 31 mars et 3 avril 2020 grâce au dispositif MoRPHEE. Trente-six patients souffrant de syndrome de détresse respiratoire aiguë (SDRA) ont été évacués durant six missions d'évacuations sanitaires collectives. Le SDRA était modéré (rapport $\mathrm{PaO}_{2} / \mathrm{FiO}_{2}>100$ et $\leq 200$ ) chez 24 patients et léger (rapport $\mathrm{PaO}_{2} / \mathrm{FiO}_{2}>200$ et $\leq 300$ ) chez

\author{
M. Beaussac \\ 160e Antenne médicale du 10e Centre médical des armées, \\ base aérienne 125 d'Istres, F-13800 Istres, France \\ B. Distinguin \\ 158e Antenne médicale du 10e Centre médical des armées, \\ base aérienne 701 de Salon-de-Provence, \\ F-13300 Salon-de-Provence, France \\ J. Turc \\ Service d'anesthésie-réanimation, \\ hôpital d'instruction des armées Desgenettes, \\ F-69003 Lyon, France \\ M. Boutonnet $(\bowtie)$ \\ Service d'anesthésie-réanimation, \\ hôpital d'instruction des armées Percy, \\ F-92140 Clamart, France \\ e-mail : mathieuboutonnet@hotmail.com \\ École du Val-de-Grâce, 1, place Alphonse-Laveran, \\ F-75005 Paris, France
}

12 patients. La durée médiane de la ventilation mécanique en soins intensifs avant le transport était de quatre jours (interquartile [IQ] : 3-5). Le rapport médian $\mathrm{PaO}_{2} / \mathrm{FiO}_{2}$ était de $180 \mathrm{mmHg}$ (IQ : 156-202). Le débit médian de perfusion de noradrénaline était de $0,08 \mu \mathrm{g} / \mathrm{kg}$ par min. Aucune complication mettant en jeu le pronostic vital n'a été signalée. En conclusion, l'évacuation sanitaire aérienne collective de patients gravement malades de Covid-19 est une solution contribuant à contrôler le niveau de saturation du système de soins au niveau national ou international.

Mots clés SDRA · Covid-19 - Évacuation aérosanitaire Médecine militaire

Abstract Since its emergence in China in late 2019, the coronavirus disease 2019 (COVID-19) outbreak has rapidly posed the risk of critical care resources overload in every affected country. The distribution of the disease among the territories is heterogeneous. Long-range inter-hospital transport of critically ill COVID-19 patients as a way to mitigate the localised pressure from overloaded intensive care units at a national or international level has not been reported yet. The aim of this article was to provide descriptive data about the six collective aeromedical evacuation of COVID19 patients performed within Europe and French national territory on March 18, 21, 24, 27, 31 and April 3, 2020. Thirty-six patients with acute respiratory distress syndrome (ARDS) were transported aboard six aeromedical evacuation missions. ARDS was moderate $\left(\mathrm{PaO}_{2} / \mathrm{FiO}_{2}\right.$ ratio $>100$ and $\leq$ $200)$ in 24 patients and mild $\left(\mathrm{PaO}_{2} / \mathrm{FiO}_{2}\right.$ ratio $>200$ and $\leq$ 300 ) in 12 patients. The median duration of mechanical ventilation in ICU before transportation was 4 days [Interquartile (IQ): 3-5]. The median $\mathrm{PaO}_{2} / \mathrm{FiO}_{2}$ ratio was $180 \mathrm{mmHg}$ (IQ: 156-202). The median norepinephrine infusion rate was $0.08 \mu \mathrm{g} / \mathrm{kg} / \mathrm{min}$. No life-threatening event was reported. Collective aeromedical evacuation of COVID-19 critically ill patients could provide a reliable solution to help control 
the burden of the disease at a national or international level. Future works may evaluate the global safety and effectiveness of aeromedical evacuations in this context.

Keywords ARDS · COVID-19 - Aeromedical evacuation · Military medicine

\section{Introduction}

Depuis son émergence en Chine à la fin de l'année 2019, l'épidémie de coronavirus 2019 (Covid-19) s'est rapidement propagée, au point d'être qualifiée le 30 janvier 2020 d'urgence sanitaire internationale [1]. La France et la majeure partie de l'Europe ont été atteintes principalement à partir de la fin du mois de février 2020 [2]. Le 11 mars 2020, l'Organisation mondiale de la santé (OMS) qualifia l'épidémie de Covid-19 de pandémie [3].

La particularité de l'infection de la Covid-19 est le nombre élevé de formes respiratoires graves avec syndrome de détresse respiratoire aiguë (SDRA), avec une augmentation rapide du nombre de cas nécessitant une prise en charge en réanimation $[4,5]$. Le risque encouru est alors la saturation des services de soins intensifs localement disponibles. Les premières réponses à l'épidémie ont inclus la réalisation de tests de diagnostic rapide et des gestes barrières (distanciation sociale, diminution des interactions, lavage des mains...) afin de limiter le taux de transmission et le nombre de nouveaux cas. Dans la mesure du possible, les établissements de soins ont augmenté leur capacité d'accueil de soins et de réanimation [6,7]. Le transport de patients entre différentes structures de soins avait déjà été envisagé dans des situations de catastrophes naturelles, mais pas dans un contexte de pandémie [8].

Le confinement a été officiellement mis en place en France le 17 mars 2020 [9]. Le même jour, 7730 cas de Covid-19 ont été confirmés, dont 699 hospitalisés en réanimation [2]. Comme dans d'autres pays touchés par l'épidémie, la répartition des cas était hétérogène en France : la majorité des cas étaient initialement dans la région Grand Est, avec une saturation des services de réanimation régionaux, pendant que d'autres régions étaient moins touchées : lors du pic épidémique (7 avril 2020), 950 des 7131 patients de réanimation étaient hospitalisés dans la région Grand Est contre 243 en Nouvelle-Aquitaine, 176 en Pays de la Loire et 133 en Bretagne [10].

Face à cette crise sanitaire inédite, le gouvernement français décida d'organiser le transport médicalisé de patients SARS-Cov-2 depuis la région Grand Est vers des régions moins saturées, afin d'adapter les ressources au besoin en soins intensifs et de lutter contre la saturation du réseau de soins du Grand Est. Le service de santé des armées (SSA) a alors été sollicité pour assurer des évacuations aériennes collectives, que nous désignerons sous le terme d'Evasan (évacuation sanitaire) collective. Un A330 MRTT (Multi Role Tanker and Transport) a été équipé des modules MoRPHEE (module de réanimation pour patient à haute élongation d'évacuation), devenant ainsi une unité de soins intensifs volante, capable de transporter des patients de réanimation sur des vols longue distance (Figs 1,2). Ainsi équipé, le MRTT permettait une MEDEVAC collective de six patients porteurs d'un SDRA lié à la Covid-19 [11]. Initialement créée pour assurer le rapatriement sanitaire des blessés de guerre depuis des territoires éloignés de la France métropolitaine, la mission MoRPHEE a réalisé l'évacuation de 36 patients SARS-Cov-2, répartis en six missions du 18 mars au 3 avril 2020. Durant cette période, 600 autres évacuations de patients ont été réalisées par voie ferroviaire ou aérienne. Le transport interhospitalier de patients sévères est reconnu comme à haut risque de complication et d'aggravation [12-14]. Des évacuations de patients en SDRA sous ventilation mécanique ont déjà été réalisées par le passé $[15,16]$, mais par le biais d'évacuations individuelles. Peu de données de littérature ont été retrouvées sur les évacuations collectives de patients en SDRA, en particulier en contexte de pandémie. Ces 36 évacuations sanitaires aéroportées interhospitalières ont donc été un véritable défi à relever.

\section{Concept MoRPHEE}

Le SSA a mis en place depuis plusieurs années le concept MoRPHEE, permettant une évacuation stratégique des blessés de guerre graves, afin de leur assurer un meilleur pronostic vital ou fonctionnel $[17,18]$. Ce concept étant

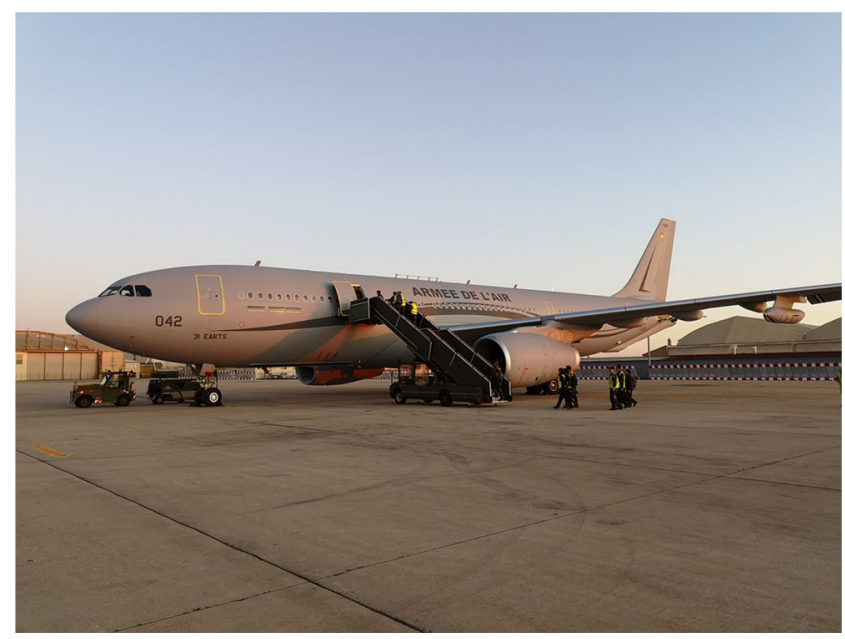

Fig. 1 Airbus A330 Phénix au parking (Crédits photos : ministère des Armées) 


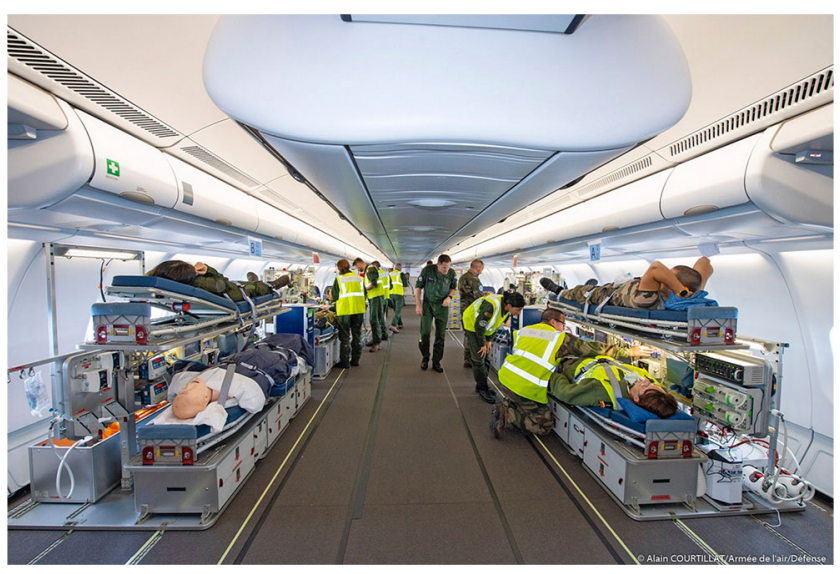

Fig. 2 Configuration MoRPHEE à bord de l'Airbus A330 Phénix (Crédits photos : ministère des Armées)

opérationnel depuis le $1^{\text {er }}$ septembre 2006, cinq missions avaient été précédemment déclenchées, permettant le rapatriement de 57 patients souffrant de traumatismes physiques graves [11]. Aucun patient en SDRA n'avait été jusqu'à présent évacué par MoRPHEE.

Le système MoRPHEE repose sur l'équipement opérationnel en quelques heures d'un vecteur aérien initialement non dédié au transport de blessés [19], en un espace médicalisé de haut niveau de soins. Avec deux configurations possibles, MoRPHEE peut transporter entre 6 et 12 patients, avec respectivement six ou quatre patients intubés-ventilés, avec à bord le matériel nécessaire pour la ventilation mécanique $\left(\right.$ LTV $^{\circledR}$ 1200, Pulmonetics System), un monitorage continu, l'administration de catécholamines, médicaments anesthésiques, d'antibiothérapie, la réalisation d'échographies et de biologie délocalisée.

L'équipe médicale d'une mission MoRPHEE comprend habituellement 11 personnels médicaux et paramédicaux du SSA, issus des hôpitaux des armées, des services médicaux des unités aéronautiques de la région Sud-Est et de l'escadrille aérosanitaire de la base aérienne de Villacoublay [21]. Elle est constituée de deux médecins anesthésistesréanimateurs, de deux médecins titulaires du brevet de médecine aéronautique, de trois infirmiers anesthésistes (IADE), de deux infirmiers (IDE) d'unités aéronautiques et de deux infirmiers convoyeurs de l'air. Une $12^{\mathrm{e}}$ place est disponible pour un personnel spécialisé en fonction du profil de la mission (médecin étranger, psychiatre, chirurgien). La présence de membres spécialisés en aéronautique permet ainsi une communication avec l'équipage naviguant et une adaptation des vols aux pathologies des patients transportés.

Le dimensionnement de cette équipe est en concordance avec les recommandations OTAN, du Stanag 3204 [22], définissant les compositions des équipes de convoyage en fonction du nombre et du degré de dépendance des patients transportés. L'ensemble de ces personnels bénéficie d'une formation spécifique à l'emploi du dispositif MoRPHEE régulièrement renouvelée, permettant l'acquisition de compétences aéronautiques spécifiques des évacuations sanitaires et leur participation à l'astreinte opérationnelle MoRPHEE permanente [21].

L'A330 MRTT utilisé durant ces Evasan a une autonomie aéronautique maximale de 10000 à $12000 \mathrm{~km}$. L'autonomie calculée en termes de durée de mission est limitée à neuf heures à cause de la consommation d'oxygène estimée (secours inclus à $1000 \mathrm{l} / \mathrm{h}$ par patient). Dans le contexte de Covid-19, un délai de 12 heures entre deux Evasan était nécessaire du fait de la décontamination de l'avion. Un seul était dédié à cette mission, afin de permettre le maintien opérationnel des autres aéronefs.

\section{Réalisation de la mission}

Dans le cadre des Evasan aériennes collectives de patients Covid19, les équipes médicales étaient composées de trois anesthésistes-réanimateurs (dont un ayant le rôle de directeur médical), deux médecins des forces spécialisés en aéronautiques, deux IADE, deux IDE des forces et trois infirmiers convoyeurs. Sur le plan infectieux (risque biologique), l'équipe a été renforcée par des spécialistes biologistes de l'Institut de recherche biomédicale des armées (IRBA) et de la cellule NRBC (nucléaire, radiologique, biologique et chimique) de la base aérienne de Cazaux.

Chaque mission était organisée en coordination avec le directeur médical des missions MoRPHEE, l'état-major opérationnel de Santé (EMOS), le Samu 68 ou 57, l'agence régionale de santé (ARS) et les chefs de réanimation de la région Grand Est, les Samu et ARS des régions accueillantes et les chefs de service de réanimation accueillants. Six patients étaient sélectionnés après discussion entre les équipes de réanimation des hôpitaux du Grand Est et en accord avec les familles, selon les critères décrits dans le tableau 1.

Les modalités pratiques (aéroports de décollage et d'arrivée, chariots élévateurs de chargement/déchargement, services de destination) étaient fixées lors de la conférence téléphonique la veille des vols. Une fois les patients identifiés et leurs services initiaux connus, l'équipe médicale MoRPHEE contactait les réanimations du Grand Est afin de recueillir les différentes données médicales, de hiérarchiser la gravité des patients et d'établir un plan de chargement et de déchargement, ainsi que la position dans l'avion afin de répartir la charge de travail en cabine, au sein des trois grandes zones de travail.

Le jour de la mission, chaque poste était identifié par l'identité du patient qu'il allait accueillir et l'ordre d'embarquement/débarquement. Le vol aller au décollage d'Istres et vers la région Grand Est (Bâle-Mulhouse ou Luxembourg) 
Tableau 1 Critères de sélection des patients éligibles au transport aérien lors des missions MoRPHEE liées à la Covid-19

\begin{tabular}{|ll|}
\hline Type de critère & Caractéristique \\
\hline Pathologie & Infection Covid-19 confirmée \\
Respiratoires & $\mathrm{PaO}_{2} / \mathrm{FiO}_{2}>120 \mathrm{mmHg}$ \\
& $\mathrm{Absence}$ de ventilation en décubitus ventral \\
Hémodynamique & Vitesse de perfusion de noradrénaline $<5 \mathrm{mg} / \mathrm{h}$ \\
Poids corporel & $<120 \mathrm{~kg}$ \\
\hline $\mathrm{PaO}_{2} / \mathrm{FiO}_{2}:$ rapport pression artérielle en oxygène sur fraction inspirée en oxygène \\
\hline
\end{tabular}

servait aux derniers préparatifs et à l'habillage du personnel en éléments de protection individuels.

L'embarquement et le débarquement des patients suivaient le plan de chargement/déchargement. Les plateformes élévatrices (loaders) établies de chaque côté de l'avion afin d'embarquer/débarquer deux patients en même temps permettaient un gain de temps dans la prise en charge.

Une fois les patients débarqués dans les ambulances de réanimation des régions d'accueil pour leur transfert en réanimation, l'équipe médicale procédait au bionettoyage (désinfection des surfaces et matériels). Leur propre déshabillage était organisé par l'équipe spécialiste NRBC pendant le reste du vol retour vers la base aérienne d'Istres. S'ensuivait un débriefing de la mission. Le matériel consommable, et notamment l'oxygène, était reconditionné et rechargé entre chaque mission.

\section{Description des Evasan}

Six Evasan aériennes collectives (36 patients évacués) ont été réalisées les 18,21, 24, 27, 31 mars et le 3 avril 2020 depuis les hôpitaux de Mulhouse $(n=16)$, Metz $(n=10)$, Colmar $(n=6)$, Thionville $(n=2)$, vers les hôpitaux de Bordeaux $(n=12)$, Toulouse $(n=6)$, Brest $(n=4)$, Marseille $(n=3)$, Toulon $(n=3)$ et Quimper $(n=2)$ en France, et vers Kiel $(n=3)$ et Lubeck $(n=3)$, en Allemagne.

L'âge médian des patients était de 64 ans (interquartile [IQ] : 49-78), avec $67 \%(n=24)$ d'hommes. Les comorbidités les plus fréquentes étaient l'hypertension artérielle $(n=18,50 \%)$, l'obésité $(n=17,47 \%)$ et le diabète $(n=$ $13,36 \%$ ). L'index de comorbidité médian de Charlson était de 1 (IQ : 0-1,25).

Tous les patients remplissaient les critères de SDRA de la classification de Berlin [20], avec 24 cas de SDRA sévères à modérés (ratio $\mathrm{PaO}_{2} / \mathrm{FiO}_{2}$ entre 100 et $200 \mathrm{mmHg}$ ) et 12 SDRA légers (ratio $\mathrm{PaO}_{2} / \mathrm{FiO}_{2}$ entre 200 et $300 \mathrm{mmHg}$ ). La durée médiane de ventilation mécanique avant l'Evasan était de quatre jours (IQ : 3-5). Cinq patients avaient bénéficié au cours de leur hospitalisation de séances de décubitus ventral, et un patient a nécessité une circulation extracorporelle deux jours avant son évacuation.

Le volume courant médian était de $6,5 \mathrm{ml} / \mathrm{kg}$ (PBW) [IQ : 6,2-7] avec une PEP de $13 \mathrm{cmH}_{2} \mathrm{O}$ (IQ : 12-14). Au cours de ces six Evasan aériennes collectives, 37 événements intercurrents eurent lieu pendant les vols nécessitant une intervention médicale chez 23 patients. Tous ont été rapidement contrôlés par l'équipe médicale à bord.

La durée médiane de vol était de 71 min (IQ : 64-74) pour une distance entre aéroports médiane de $800 \mathrm{~km}$ [717-830]. La durée médiane de transport aérien (de l'embarquement des patients jusqu'à leur débarquement) était de $185 \mathrm{~min}$ (IQ : 145-198). Pour chaque MEDEVAC collective, le temps d'embarquement/débarquement des patients était compris entre 60 et $100 \mathrm{~min}$, avec une durée de conditionnement de chaque patient d'environ 15 à $25 \mathrm{~min}$.

\section{Analyse}

Au total, ces six missions MoRPHEE ont permis de transférer 36 patients de réanimation, atteints de SDRA lié à la Covid-19. Elles ont été les premières Evasan à être réalisées et se sont appuyées sur l'expertise, la logistique et l'organisation du SSA et de l'armée de l'air, grâce à un système éprouvé, des équipes entraînées et d'alerte 365 jours par an.

Aucun décès en cours de transfert ou dans les heures suivantes n'est intervenu. Cela est un argument pour attester de la pertinence des critères de sélection choisis avant le transport et de la qualité des soins délivrés en vol.

Les limites de la mission se sont révélées être principalement liées aux exigences aéroportuaires, qui nécessitaient la présence de plateformes élévatrices pour l'embarquement et le débarquement des patients. De telles plateformes ne se trouvent pas disponibles sur l'ensemble des infrastructures aéroportuaires, ce qui a amené à choisir des aéroports internationaux dotés de tels dispositifs pour décoller et atterrir lors des missions. La mise en place d'autres vecteurs d'évacuation tel l'Airbus A400M qui ne nécessite pas de telles plateformes permettra à l'avenir de pallier ce problème et 
de se poser ou de décoller à partir de pistes plus sommaires. À ce titre, le retour d'expérience des missions de l'A400M en Guyane en juillet 2020 sera instructif.

\section{Conclusion}

Ces Evasan aériennes collectives de patients en SDRA dans le contexte épidémique de la pandémie de Covid-19 ont été les premières à avoir été réalisées en France et en Europe. Dans un contexte de saturation des réseaux de soins, les Evasan aériennes collectives de patients de réanimation sont réalisables et contribuent à la meilleure prise en charge du plus grand nombre de patients, à un niveau national ou international.

Liens d'intérêts : les auteurs déclarent ne pas avoir de liens d'intérêts.

\section{Éléments remarquables}

- Solidité et rapidité de mise en œuvre du dispositif MoRPHEE (fiabilité des dispositifs et des organisations/adaptabilité et professionnalisme d'équipes formées et entraînées au dispositif)

- Coordination opérationnelle de la mission impliquant l'Étatmajor opérationnel santé du Service santé des armées (SSA), les agences régionales de santé (ARS) et services de réanimation de départ et d'arrivée, le directeur médical de la mission MoRPHEE, les représentants des intervenants civils et militaires impliqués dans le transport sur les petites boucles d'amont et d'aval (Samu, BMPM) ainsi que les structures aéroportuaires - Efficacité des chaînes aéronautique (armée de l'Air), médicale et de ravitaillement sanitaire (service de santé des armées)

- Pertinence des critères de sélection des patients éligibles à un transfert

\section{Pistes d'amélioration}

- Mise en place d'un dispositif informatique permettant le partage de données médicales sécurisé, fiable et respectant la législation et les règles en vigueur (Règlement général sur la protection des données : RGPD)

\section{Références}

1. World Health Organization (2005) Statement on the second meeting of the International Health Regulations Emergency Committee regarding the outbreak of novel coronavirus (2019-nCoV). https://www.who.int/news290 room/detail/30-01-2020-statementon-the-second-meeting-of-the-internationalhealth-regulations(2005)-emergency-committee-regarding-the-outbreak-of-novel coronavirus-(2019-ncov) (Dernier accès le 27 mars 2020)

2. Gouvernement de la République française (2020) Cartes et données. https://www.gouvernement.fr/info-coronavirus/carte-et-donnees (Dernier accès le 28 mars 2020)

3. World Health Organization (2020) Coronavirus disease 2019 (Covid-19) Situation Report - 11 March. https://www.who.int/ $\mathrm{dg} /$ speeches/detail/who-director-general-s-opening-remarksat-themedia-briefing-on-covid-19-11-march-2020 (Dernier accès le 28 mars 2020)

4. Huang C, Wang Y, Li X, et al (2020) Clinical features of patients infected with 2019 novel coronavirus in Wuhan, China. Lancet 395:497-506

5. Zhou F, Yu T, Du R, et al (2020) Clinical course and risk factors for mortality of adult in patients with Covid-19 in Wuhan, China: a retrospective cohort study. Lancet 28:1054-62

6. Mattiuzzi C, Lippi G (2020) Which lessons shall we learn from the 2019 novel coronavirus outbreak? Ann Transl Med 8:48
7. The Covid-19 AP-HP Group (2020) Assistance publique-Hôpitaux de Paris' response to the Covid-19 pandemic. Lancet 395:1760-1

8. King MA, Niven AS, Beninati W, et al (2014) Evacuation of the ICU: care of the critically ill and injured during pandemics and disasters: CHEST consensus statement. Chest 146:44-60

9. Ministère des solidarités et de la santé (2020) Coronavirus : information aux soignants. https://solidarites-sante.gouv.fr/soins-et maladies/maladies/maladies-infectieuses/coronavirus/ (Dernier accès le 28 mars 2020)

10. Santé Publique France (2020) Covid-19 : Point épidémiologique hebdomadaire du 9 avril 2020. https://www.santepubliquefrance. fr/content/download/243797/2565951 (Dernier accès le 28 mars 2020)

11. Borne M, Tourtier JP, Ramsang S, et al (2012) Collective air medical evacuation: the French tool. Air Med J 31:124-8

12. Strauch U, Bergmans DC, Winkens B, Roekaerts PM (2015) Short-term outcomes and mortality after interhospital intensive care transportation: an observational prospective cohort study of 368 consecutive transports with a mobile intensive care unit. BMJ Open 28:e006801

13. Blecha S, Dodoo-Schittko F, Brandstetter S, et al (2018) Quality of inter-hospital transportation in 431 transport survivor patients suffering from acute respiratory distress syndrome referred to specialist centers. Ann Intensive Care 8:5 
14. Arthur KR, Kelz RR, Mills AM, et al (2013) Interhospital transfer: an independent risk factor for mortality in the surgical intensive care unit. Am Surg 79:909-13

15. Zimmermann M, Bein T, Philipp A, et al (2006) Interhospital transportation of patients with severe lung failure on pumpless extracorporeal lung assist. Br J Anaesth 96:63-6

16. Schmitt J, Boutonnet M, Goutorbe P, et al (2020) Acute respiratory distress syndrome in the forward environment. Retrospective analysis of ARDS cases among French Army war casualties. J Trauma Acute Care Surg 89(Suppl 2):S207-12

17. Grasser L, De Rudnicki S, Tourtier JP, Auroy Y (2011) MoRPHEE : un système d'évacuation sanitaire aérienne stratégique collective. Rev Med Aero Spat 52:144-9
18. Grasser L, De Rudnicki S, Puidupin M, et al (2013) Évacuations stratégiques aériennes collectives des blessés par mission MoRPHEE. Rev Med Urg 84:1-15

19. Borne M, Derain P (2007) Avions vecteurs d'évacuations sanitaires aériennes. Reanoxyo 21:20-1

20. ARDS Definition Task Force, Ranieri VM, Rubenfeld GD, et al (2012) Acute respiratory distress syndrome: the Berlin definition. JAMA 307:2526-33

21. Boutonnet M, Pasquier P, Raynaud L, et al (2017) Ten years of en route critical care training. Air Med J 36:62-6

22. NATO Standardization Agency (2010) Stanag 3204 AMD (Edition 8). Aeromedical evacuation. Brussels 